\title{
Controle LQR Aplicado ao Movimento da Articulação do Joelho de Pacientes Paraplégicos
}

\section{LQR control applied to the movement of the knee of paraplegic patients}

\author{
Tiago C. de Oliveira'; Ruberlei Gaino²; Márcio R. Covacic²; Marcelo C. M. Teixeira ${ }^{3}$; \\ Aparecido A. de Carvalho ${ }^{3}$
}

\section{Resumo}

O objetivo desse artigo foi projetar um controlador linear quadrático em malha fechada, aplicado ao movimento da articulação do joelho de pacientes paraplégicos. A modelagem da dinâmica do modelo do paciente paraplégico foi realizada através da representação por variáveis de estado. Linearizandose o modelo com o ângulo da articulação do joelho em torno de $30^{\circ}$, foi possível realizar simulações que demonstraram a eficiência do controlador LQR, possuindo um índice de desempenho que deve ser minimizado para obter sua otimalidade.

Palavras-chave: Regulador Linear Quadrático, Paraplégico, Eletroestimulação, Engenharia de Reabilitação.

\begin{abstract}
The aim of this article was to design a closed loop linear quadratic controller applied to the movement of the knee joint in paraplegic patients. The modeling of the dynamic model of the paraplegic patient was performed by representation by state variables. Linearizing the model with knee join angle around $30^{\circ}$, it was possible to perform simulations that demonstrated the effectiveness of LQR controller that having a performance index to be minimized to obtain their optimality.
\end{abstract}

Key words: Linear Quadratic Regulator, Paraplegic, Electrical Stimulation, Rehabilitation Engineering.

1 Aluno de mestrado do Departamento de Engenharia Elétrica, Universidade Estadual de Londrina; tiagoco@msn.com

2 Docente do Departamento de Engenharia Elétrica da Universidade Estadual de Londrina - Deel-uel; rgaino@uel.br, marciocovacic@uel.br

3 Docentes do Departamento de Engenharia Elétrica da Universidade Estadual Paulista-“Júlio de Mesquita Filho"-FEIS-UNESP; marcelo@dee.feis.unesp.br, aac@dee.feis.unesp.br 


\section{Introdução}

O estudo do controle do movimento de pacientes paraplégicos com o uso de estimulação elétrica neuromuscular é um assunto relevante na engenharia de reabilitação (Ferrarin e Pedotti, 2000; Gaino et al., 2008; Gaino, 2009; Sanches et al., 2010; Gaino et al., 2011). A reabilitação em malha fechada utilizando estimulação elétrica funcional (FES Functional Electrical Stimulation) é uma forma de tratamento que utiliza a corrente elétrica para provocar a contração de músculos (Crago, Mortimer e Pecham, 1980). Na Figura 1, adaptada de (Silva, 2007), ilustra-se o diagrama do sistema de controle, para uma compreensão melhor do objetivo.

No Canadá, um hemiplégico recuperou a sensibilidade e o movimento do membro inferior direito após ser submetido por longos períodos a estímulos elétricos. Nos Estados Unidos, um homem paralítico há 17 anos, depois de um tratamento fisioterápico de um ano, levantou-se da cadeira e deu vários passos pela sala onde estava. Na Alemanha, alguns pacientes, após um tratamento fisioterápitico, começaram a recuperar a capacidade de andar depois de meses de tratamento intensivo. Um destes pacientes, que era paraplégico, recuperou quase totalmente os movimentos das pernas depois de um ano de tratamento, sendo capaz de caminhar com um andador, e com alguma ajuda foi capaz até de subir degraus de uma escada. No Brasil, Cliquet e sua equipe conseguiram fazer com que um rapaz voltasse a caminhar apoiado num andador, após sessões de estímulo neuromuscular (Martin, 1999). Embora sua técnica seja de malha aberta, com neuro aplicado ao músculo quadríceps.

O controle em malha fechada requer técnicas de controle eficientes, pois a fisiologia humana e os modelos musculares são muito complexos (Hill, 1938; Huxley, 1957). Estudos de modelos matemáticos e projetos com controle clássico digital foram utilizados em (Chizeck et al., 1983) e (Crago, Mortimer e Peckam, 1980).
O que se pode observar é a dificuldade de obter bons resultados utilizando a teoria de sistemas lineares. (Abbas e Chizeck, 1995) e (Chang et al, 1997) tiveram bons resultados utilizando redes neurais, pois, sendo o músculo muito complexo, os parâmetros do controle devem ser variáveis. (Chang e Wang, 1998) e (Riener e Fuhr, 1998), implementam lógica Fuzzy Mamdani em pacientes paraplégicos. A conclusão é que, de fato, a técnica não-linear é a mais eficiente.

A Estação de Controle é constituída essencialmente por um computador, sendo integrada a uma rede local por meio de comunicação sem fio, contendo um programa que possui uma rotina para gerenciar a aquisição de sinais produzidos pelo módulo de monitoramento e um algoritmo de controle.

A FES, pelo princípio de funcionamento e pelos resultados obtidos, produz contração muscular semelhante à contração gerada por um estímulo enviado pelo Sistema Nervoso Central - SNC. Sua aplicação em tratamentos fisioterápicos de pacientes paraplégicos tem eficácia comprovada (Handa et al., 1990; Chen et al., 1997; Yu et al., 1999).

Como mostrado na Figura 2, quando é aplicada uma diferença de potencial entre dois eletrodos, há circulação de corrente no interior do tecido, que por ser predomi-nantemente aquoso, dá-se pelo movimento ordenado de íons (Silva, 2007).

A amplitude/intensidade e a duração do estímulo elétrico devem ter um valor mínimo para evocar um PA (Potencial de Ação), isto é, acima de um limiar. A proximidade do eletrodo com a fibra nervosa, seu diâmetro, entre outras características, determinam a velocidade de recrutamento, bem com o número de fibras recrutadas (Robinson, 2001). O projeto do neuroestimulador com FES em malha fechada com o paciente paraplégico foi estudado mais recentemente em (Faria, 2006) e (Silva, 2007). Recentemente, em (Kozan, 2012) e (Sanches, 2013), foram realizadas as primeiras dissertações e teses do país envolvendo controle, neuroestimulador e 
pacientes paraplégicos em malha fechada. Pelo conhecimento dos autores em 2014 (Sanches et al., 2014), está sendo publicado o primeiro artigo do país com aplicação em paciente paraplégico. A proposta deste controle pode ser implementada, elaborando os algoritmos dessa técnica e inserindo na estação de controle, como mostrado na Figura 2.

A eletroestimulação utilizada adequadamente pode auxiliar na recuperação de um paciente, atuando, por exemplo, no sistema circulatório, aumentando a circulação de sangue no membro paralisado e contendo a atrofia muscular (Crameri et al., 2004).
A motivação para a realização deste trabalho concentra-se na pouca informação existente no Brasil sobre grupos de pesquisas com aplicação de FES em pacientes paraplégicos com realimentação em malha fechada. No Congresso Brasileiro de Engenharia Biomédica (CBEB), realizado em Salvador-BA em 2008, os únicos trabalhos publicados sobre reabilitação de pacientes paraplégicos com FES em malha fechada foram realizados por (Gaino et al., 2008) e (Prado et al., 2008), com foco na solução dos problemas vivenciados por pacientes paraplégicos e hemiplégicos com o objetivo de melhorar a qualidade de suas vidas.

Figura 1 - Ilustração do hardware utilizado para controle, adaptado de (Silva, 2007).

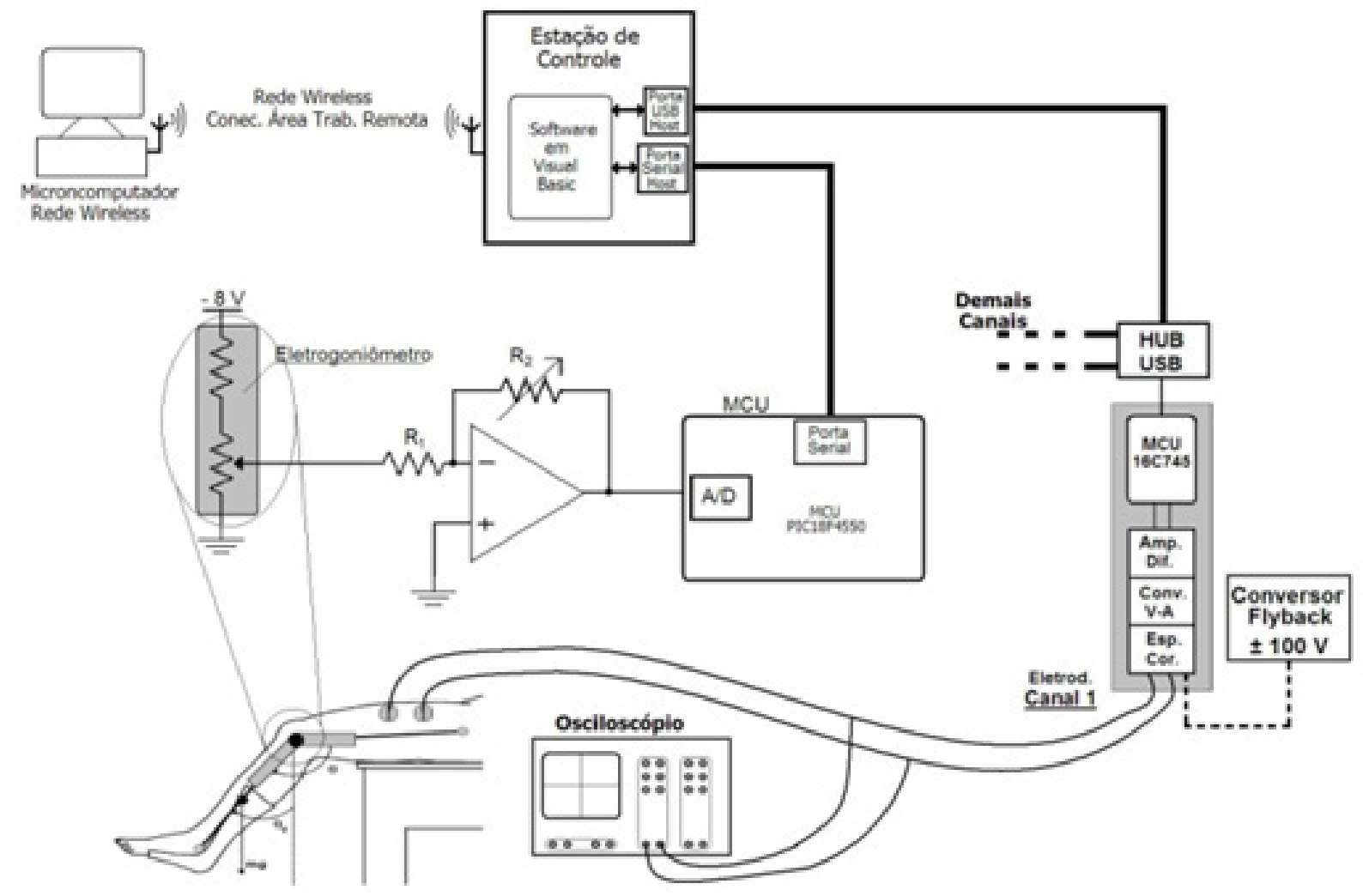


Figura 2 - Estimulação elétrica do nervo por meio de eletrodos externos, adaptado de (Robinson, 2001).

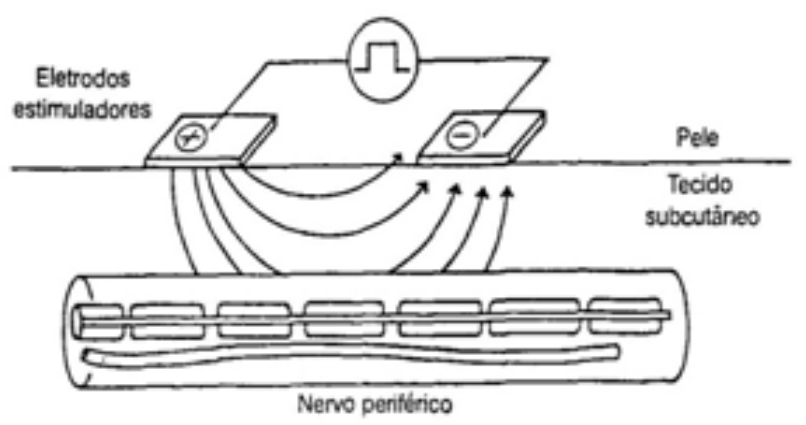

O uso de modelos matemáticos que representem uma situação aproximada de um determinado sistema possibilita a investigação de seu comportamento, por meio de computadores, sem que haja a necessidade de sua construção.

Este trabalho consiste no desenvolvimento, em ambiente de simulação computacional, de um controlador linear para posicionar a perna do paciente paraplégico em um ângulo desejado. As equações matemáticas do paciente paraplégico constituem uma dinâmica não-linear (Gaino, 2009), mas o sistema é linearizado em torno do ponto de operação de interesse e é projetado um controlador que imponha as restrições de desempenho (Gaino et al., 2007). Métodos lineares foram implementados com sucesso por (Crago, Mortimer e Pecham, 1980).

Poucas aplicações de teoria de controle ótimo foram encontradas na literatura abordando o estudo do movimento a articulação do joelho do paciente paraplégico. Devido a isto, os autores fizeram o estudo em simulação para observar o comportamento deste sistema linear proposto. Projetos de sistemas lineares foram publicados em (Gaino et al., 2008) e (Prado et al., 2008). Modelos não-lineares mais complexos foram publicados em congressos e periódicos (Teixeira et al. 2006a; Teixeira et al. 2006b; Gaino et al., 2011; Covacic et al., 2012) considerando pontos de operação de $30^{\circ}$ e $60^{\circ}$.
Os resultados deste artigo, com modelo linearizado trabalhando em torno do seu ponto de operação, mostram também uma viabilidade com boas perspectivas para implementação. Estudos do grupo já produziram o neuroestimulador para implementação dos algoritmos de controle que estão sendo gerados pelo grupo FEIS-UNESP e UEL, seja o sistema linear ou não-linear (Silva, 2007).

O eletroestimulador implementado é um equipamento compacto (Figura 1), com conexão USB, de fácil manuseio, e pode ser conectado não somente à estação de controle, mas a qualquer outro computador. A Figura 3 mostra a forma de onda produzida pelo neuroestimulador (Silva, Carvalho 2007).

Figura 3 - Forma de onda na entrada do espelho de corrente do Eletroestimulador, gravada pelo osciloscópio (Gaino et al., 2007).

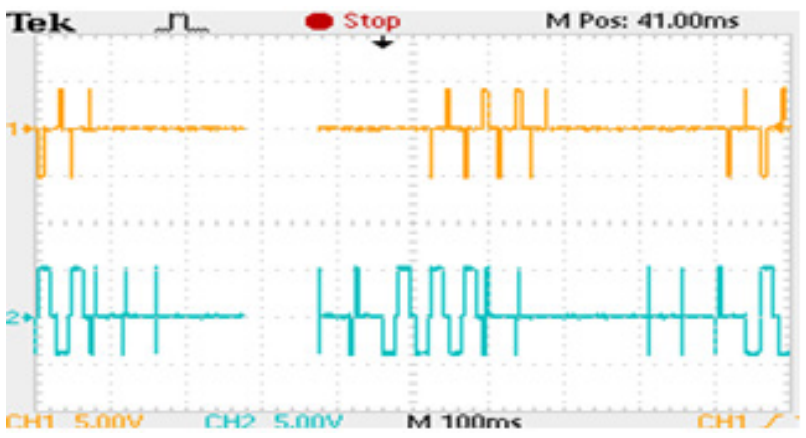

O presente trabalho propõe, em simulação no software Matlab, o uso do regulador linear quadrático (Linear Quadratic Regulator - LQR) (Ogata, 2010) para controle da variação angular da articulação do joelho de pacientes paraplégicos, com estímulos elétricos no músculo quadríceps, trabalhando com uma referência desejada, partindo do repouso e estabilizando-se no ponto desejado.

Como o modelo utilizado é não-linear (Teixeira et al. 2006a; (Teixeira et al. 2006b; Gaino, 2009), torna-se necessário linearizar o mesmo para que 
seja possível projetar o controlador linear LQR para um determinado ponto operação, neste caso $30^{\circ}$.

$\mathrm{Na}$ literatura especializada com uso de controle LQR em engenharia reabilitação, foram encontrados vários trabalhos. Em (Iqbal e Mughal, 2007), foi desenvolvido um modelo sagital de vários segmentos com três graus de liberdade, incluindo a rotação no tornozelo, joelho e quadril. Foi proposto um controlador LQR ótimo análogo ao sistema nervoso central de coordenação da postura e movimento. Em (Peterson e Chizeck, 1987), foi aplicada a teoria do LQR sobre um sistema formado por dois músculos, atuando sobre uma carga externa, linear de segunda ordem, em uma configuração agonista-antagonista, com o objetivo de ativar esses músculos por meio de impulsos elétricos para obter uma determinada posição na trajetória da carga. Em (Matjacic e Bajd, 1998), foi proposto um modelo baseado na integração das atividades voluntárias e reflexas do segmento corporal neurologicamente intacto, ao controle da resistência dos tornozelos por meios mecânicos artificiais. Neste estudo foi aplicado FES de cadeia fechada sobre um modelo matemático, utilizando o LQR para o cálculo do sinal de controle.

A literatura mostra poucos trabalhos publicados com uso do LQR, no caso de reabilitação de pacientes paraplégicos, e nosso artigo evidencia a utilização de uma técnica de controle já consolidada pela literatura (Ogata, 2010; Kailath, 1980) na realização de pequenos movimentos na articulação no joelho.

\section{Regulador Linear Quadrático (LQR)}

A técnica de controle LQR representa uma solução de controle intermediária, entre as técnicas de controle mais simples (Proporcional Integral e Derivativo - PID) e as mais complexas (Preditivas), sob o ponto de vista de projeto e equacionamento (Delatore, 2011).
Através de um estado inicial qualquer, o problema do regulador ótimo é encontrar o vetor $\boldsymbol{u}(\boldsymbol{t})$ que realize a transferência de um estado para outra região do espaço de estado desejada. O desempenho desejado pode ser formulado diretamente em termos de índices de desempenho no domínio do tempo. Os sistemas que são ajustados de modo a fornecer um índice de desempenho mínimo são freqüentemente chamados de sistemas de controle ótimo (Rosa Filho, 2011).

A planta do regulador linear quadrático invariante no tempo é dada pelo sistema genérico, descrito por equações de estados:

$$
\begin{aligned}
& \dot{x}(t)=A x(t)+B u(t), \\
& y(t)=C x(t)+D u(t),
\end{aligned}
$$

sendo $\boldsymbol{A}, \boldsymbol{B}$ e $\boldsymbol{C}$ matrizes constantes de dimensões $\boldsymbol{n} \boldsymbol{x} \boldsymbol{n}, \boldsymbol{n} \boldsymbol{x} \boldsymbol{l}$ e $\mathbf{1} \boldsymbol{x} \boldsymbol{n}$ respectivamente, $\boldsymbol{D}$ um constante escalar, $\boldsymbol{x}$ o vetor de estado do modelo, $\boldsymbol{u}$ o sinal de controle e $\boldsymbol{y}$ o sinal de saída do sistema (Ogata, 2010).

A lei de controle de realimentação é definida como:

$$
u(t)=-\boldsymbol{K} x(t)
$$

sendo $\boldsymbol{K}$ a matriz de realimentação de estados. Substituindo (3) em (1), é possível obter a resposta em malha fechada,

$$
\dot{x}(t)=(A-B K) x(t) .
$$

É possível obter a otimalidade do controle LQR minimizando-se o índice de desempenho dado por:

$$
I=\int_{0}^{\infty}\left[x(t)^{T} Q x(t)+u(t)^{T} \boldsymbol{R} u(t)\right] d t
$$


sendo $\boldsymbol{Q}$ uma matriz hermitiana definida positiva (ou semidefinida positiva) ou real simétrica e $\boldsymbol{R}$ é uma matriz hermitiana definida positiva ou real simétrica.

As matrizes $\boldsymbol{Q}$ e $\boldsymbol{R}$ determinam a importância relativa do erro e do consumo de energia. A matriz $\boldsymbol{Q}$ representa a ponderação dos estados e $\boldsymbol{R}$ é a matriz de ponderação das entradas. Se os elementos da matriz $\boldsymbol{K}$ forem determinados de modo a minimizar o índice de desempenho, então $\boldsymbol{u}(t)=-\boldsymbol{K} \boldsymbol{x}(t)$ é ótimo para qualquer estado inicial $x(0)$ (Ogata, 2010).

Considerando-se uma matriz $\boldsymbol{K}$ que torna o sistema (4) assintoticamente estável, e substituindose (3) em (5), o índice de desempenho é dado por,

$$
\begin{gathered}
I=\int_{0}^{\infty}\left(x^{T} Q x+x^{T} K^{T} R K x\right) d t, \\
I=\int_{0}^{\infty} x^{T}\left(Q+K^{T} R K\right) x d t,
\end{gathered}
$$

onde a dependência em $\boldsymbol{t}$ de $\boldsymbol{x}(\mathrm{t})$ foi suprimida por simplicidade da exposição que se segue.

Considere a existência de uma equação diferencial exata tal que:

$$
x^{T}\left(Q+K^{T} R K\right) x=-\frac{d}{d t}\left(x^{T} P x\right)
$$

sendo $\boldsymbol{P}$ uma matriz hermitiana definida positiva. Considerando (4), o lado direito de (8) é dado por:

$$
\begin{aligned}
& -\frac{d}{d t}\left(x^{T} P x\right)=\dot{x}^{T} P x-x^{T} P \bar{x} \\
& =-x^{T}\left[(A-B K)^{T} P+P\right]
\end{aligned}
$$

Substituindo (9) em (8), tem-se:

$$
\begin{aligned}
& x^{T}\left(\boldsymbol{Q}+\boldsymbol{K}^{T} \boldsymbol{R} \boldsymbol{K}\right) x \\
& =-x^{T}\left[(A-B K)^{T} \boldsymbol{P}+\boldsymbol{P}((A-B K)] x\right.
\end{aligned}
$$

Assim, de (9) e (10), obtém-se:

$$
\left(Q+K^{T} R K\right)=-\left[(A-B K)^{T} P+P(A-B K)\right]
$$

Se o sistema (4)for estável, então existirá uma matriz $P$ definida positiva que satisfaz (11). Portanto, o índice de desempenho será dado por:

$I=\int_{0}^{\infty} x^{T}\left(Q+K^{T} R K\right) x d t=-\left.x^{T} P x\right|_{0} ^{\infty}=-x^{T}(\infty) P x(\infty)$

Como o sistema (4) é assintoticamente estável, $x(\infty) \rightarrow 0$ e, portanto,

$$
I=x^{T}(0) P x(0)
$$

Suponha agora que $\boldsymbol{R}$ seja uma matriz hermitiana definida positiva ou real simétrica, definida como:

$$
R=T^{T} T
$$

sendo $\boldsymbol{T}$ uma matriz não-singular. Substituindo-se (14) em (11), tem-se:

$\left(A^{T}-K^{T} B^{T}\right) P+P(A-B K)+Q+K^{T} T^{T} T K=0$

que pode ser reescrita como:

$A^{T} P+P A+\left[T K-\left(T^{T}\right)^{-1} B^{T} P\right]^{T}\left[T K-\left(T^{T}\right)^{-1} B^{T} P\right]$

$-P B R^{-1} B^{T} P+Q=0$

Para minimizar I em relação a $K$, deve-se minimizar a expressão:

$$
x^{T}\left[T K-\left(\boldsymbol{T}^{T}\right)^{-1} \boldsymbol{B}^{T} \boldsymbol{P}\right]^{T}\left[T K-\left(\boldsymbol{T}^{T}\right)^{-1} \boldsymbol{B}^{T} \boldsymbol{P}\right] x
$$

em relação a $\boldsymbol{K}$. A solução ocorre quando a expressão acima é igual a zero, isto é:

$$
T K=\left(T^{T}\right)^{-1} B^{T} P
$$

ou seja:

$$
K=T^{-1}\left(T^{T}\right)^{-1} B^{T} P=R^{-1} B^{-T} P
$$


Portanto, de acordo com (Rosa Filho, 2011), a matriz ótima $\boldsymbol{K}$ é expressa por:

$$
\boldsymbol{K}=-\boldsymbol{R}^{-1} \boldsymbol{B}^{T} \boldsymbol{P},
$$

sendo $\boldsymbol{P}$ uma matriz simétrica definida positiva, ou seja, $\boldsymbol{P}=\boldsymbol{P}^{T}>\boldsymbol{O}$.

Assim, a lei de controle ótimo do problema de controle quadrático ótimo, quando o índice de desempenho é dado por (5), é linear e é dada por:

$$
\boldsymbol{u}(t)=-\boldsymbol{K} \boldsymbol{x}(t)=\boldsymbol{R}^{-1} \boldsymbol{B}^{T} \boldsymbol{P} \boldsymbol{x}(t) .
$$

A matriz $\boldsymbol{P}$ deve satisfazer a equação matricial reduzida de Riccati,

$$
A^{T} P+P A-P B R^{-1} B^{T} P+Q=0 .
$$

\section{Modelo matemático do movimento da articulação do joelho do paciente paraplégico}

O modelo matemático em estudo foi adaptado de (Ferrarin e Pedotti, 2000) para variáveis de estado e publicado em diversos congressos e periódicos (Teixeira et al., 2006a; Teixeira et al., 2006b; Gaino, 2009; Gaino et al., 2011; Covacic et al., 2012). Da teoria de estabilidade segundo Lyapunov, foi necessário efetuar uma troca de variáveis para transladar o novo ponto de equilíbrio para a origem (Gaino, 2009).

Para aplicar o controlador LQR nesse modelo, que é não-linear, foi necessário primeiramente linearizá-lo em torno do ponto de interesse, $30^{\circ}$. As variáveis de interesse são $\theta, \dot{\theta}$ e $M_{a}$ (conforme descrição a seguir), o que difere das consideradas para o modelo não-linear.

O modelo adaptado (Ferrarin e Pedotti, 2000) relaciona a largura do pulso aplicado com o torque gerado na articulação do joelho. A perna deve voltar à posição de repouso com a retirada da estimulação no músculo mencionado (Gaino, 2009).
Na modelagem, (Ferrarin e Pedotti, 2000), considerou-se o membro inferior como uma cadeia cinemática aberta composta de dois segmentos rígidos: a coxa, e o complexo canela-pé, conforme mostra a Figura 4.

Figura 4 - Esquema de representação da perna adaptado de Ferrarin e Pedotti (Ferrarin e Pedotti, 2000)

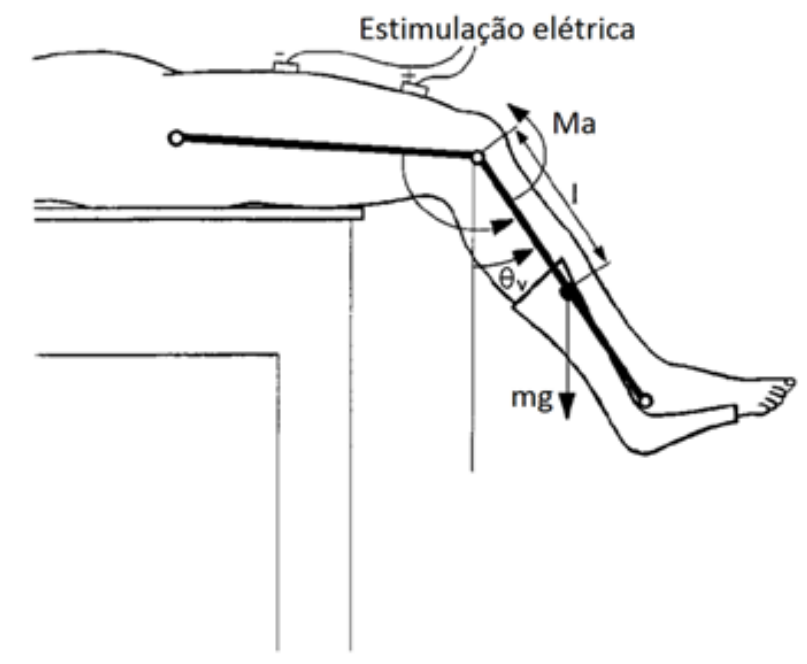

O equilíbrio dinâmico desses componentes em torno da junção do joelho é representado por :

$$
M_{i}=M_{g}+M_{s}+M_{d}+M_{a}
$$

sendo que:

$M_{i}$ é o coeficiente inercial;

$M_{g}$ é o coeficiente gravitacional;

$M_{s}$ é o torque devido ao componente de rigidez;

$M_{d}$ é coeficiente de amortecimento; e

$M_{a}$ é o torque ativo do joelho produzido pela estimulação elétrica.

Desta forma, o equilíbrio dinâmico também pode ser expresso pela equação diferencial não-linear de segunda-ordem (Ferrarin e Pedotti, 2000): 
$J \ddot{\theta}_{v}=-m g l \operatorname{sen}\left(\theta_{v}\right)+M_{s}+B \dot{\theta}+M_{a}$,

sendo que:

$J$ é o momento inercial do complexo de canelapé;

$\theta$ é o ângulo comum do joelho (ângulo entre a canela e a coxa no plano sagital);

$\dot{\theta}$ é a velocidade angular comum do joelho;

$\theta_{v}$ é o ângulo da canela (ângulo entre a canela e o sentido vertical no plano sagital);

$\ddot{\theta}_{v}$ é a aceleração angular da canela;

$m$ é a massa do complexo canela-pé;

$g$ é a aceleração gravitacional;

$l$ é a distância entre o joelho e o centro da massa do complexo canela-pé;

$B$ é o coeficiente de atrito viscoso.

Com relação ao componente da rigidez, a seguinte expressão foi considerada:

$$
M_{s}=-\lambda e^{-E \theta}(\theta-\omega),
$$

sendo $\lambda$ e $E$ coeficientes do termo exponencial e $\varpi$ o ângulo elástico de repouso do joelho.

O sinal negativo é devido à escolha do torque do extensor como positivo. Nessas fórmulas, o fator exponencial é responsável pelo comportamento não-linear da elasticidade do joelho. Foi verificado que o torque ao qual o músculo estará sujeito, $M_{a}$, e a largura dos pulsos da estimulação elétrica, $P$, podem ser relacionados, adequadamente, pela função de transferência a seguir:

$$
H(s)=\frac{M_{a}(s)}{P(s)}=\frac{G}{1+s \tau},
$$

sendo os valores de $\tau$ e de $G$ apresentados em (Ferrarin e Pedotti, 2000).

(Ferrarin e Pedotti, 2000) utilizaram a identificação de sistemas (Aguirre, 2000), para encontrar (26). Vários estudos demonstraram a dificuldade de se obter um modelo que represente o movimento sob contração muscular e sob a condição do estímulo artificial aplicado (Hill, 1938; Huxley 1957; Hatze, 1981; Winter, 1990; Herzog e Nigg, 1999).

$\mathrm{Na}$ Tabela 1, são apresentados valores antropométricos de um paciente paraplégico obtidos de maneira experimental por (Ferrarin e Pedotti, 2000).

Tabela 1 - Grandezas Antropométricas do Paciente (Ferrarin e Pedotti, 2000).

\begin{tabular}{l|l}
\hline$J$ & $0.362\left[\mathrm{Kgm}^{2}\right]$ \\
$m$ & $4.37[\mathrm{Kg}]$ \\
$l$ & $23.8[\mathrm{~cm}]$ \\
$B$ & $0.27[\mathrm{Nms} / \mathrm{rad}]$ \\
$\lambda$ & $41.208[\mathrm{Nm} / \mathrm{d}]$ \\
$E$ & $2.024[1 / \mathrm{rad}]$ \\
$\omega$ & $2.918[\mathrm{rad}]$ \\
$\tau$ & $0.951[\mathrm{~s}]$ \\
$G$ & $42500[\mathrm{Nm} / \mathrm{s}]$ \\
\hline
\end{tabular}

Substituindo (25) em (24), e considerando $\theta=\theta_{v}+\frac{\pi}{2}$ obtém-se,

$\ddot{\theta}_{v}=\frac{1}{J}\left[-m g l \operatorname{sen}\left(\theta_{v}\right)\right.$

$$
\left.-\lambda e^{-E \theta v} e^{-E \frac{\pi}{2}}\left(\theta_{v}+\frac{\pi}{2}-\varpi\right)-B \dot{\theta}_{v}+M_{a}\right]
$$


Como, no ponto de operação $\theta_{v 0}=30^{\circ}$, as derivadas $\dot{\theta}_{v}$ e $\ddot{\theta}_{v}$ são nulas, obtém-se:

$$
\begin{gathered}
M_{a 0}=m g l \operatorname{sen}\left(\theta_{v 0}\right) \\
+\lambda e^{-E \theta 0_{0}} e^{-E \frac{\pi}{2}}\left(\theta_{v 0}+\frac{\pi}{2}-\varpi\right)=4.6068[\mathrm{Nm}] .
\end{gathered}
$$

Então, de (26):

$$
(1+s \tau) M_{a}(s)=G P(s)
$$

Passando (29) para o domínio do tempo, obtémse:

$$
\tau \dot{M}_{a}+M a=G P
$$

Definindo as variáveis de estado, $\theta_{v}, \dot{\theta}_{v}$ e $M_{a}$ na forma:

$$
\begin{gathered}
\theta_{v}=x_{1}, \\
\dot{\theta}_{v}=\dot{x}_{1}=x_{2} \\
M_{a}=x_{3},
\end{gathered}
$$

e substituindo (31) em (27), encontra-se:

$$
\begin{aligned}
J x_{2} & =\left[-\frac{1}{x_{1}} m g l \operatorname{sen}\left(x_{1}\right)\right. \\
& \left.-\frac{1}{x_{1}} \lambda e^{-E x_{1}} e^{-E \frac{\pi}{2}}\left(x_{1}+\frac{\pi}{2}-\omega\right)\right] x_{1}-B x_{2}+x_{3} .
\end{aligned}
$$

Resolvendo (30) pela transformada de Laplace e substituindo (31) em (30), obtém-se,

$$
\dot{x}_{3}=-x_{3}+G P
$$

Escrevendo (32) e (33) na forma matricial, obtém-se uma forma matricial, para projeto de controladores lineares:

$$
\left[\begin{array}{l}
\dot{x}_{1}(t) \\
\dot{x}_{2}(t) \\
\dot{x}_{3}(t)
\end{array}\right]=\left[\begin{array}{ccc}
0 & 1 & 0 \\
\tilde{f}_{21}\left(x_{1}(t)\right) & -\frac{B}{J} & \frac{1}{J} \\
0 & 0 & -\frac{1}{\tau}
\end{array}\right]\left[\begin{array}{l}
x_{1}(t) \\
x_{2}(t) \\
x_{3}(t)
\end{array}\right]+\left[\begin{array}{c}
0 \\
0 \\
\frac{G}{\tau}
\end{array}\right] P
$$

sendo $\tilde{f}_{21}\left(x_{1}(t)\right)$ uma não-linearidade do sistema representada por:

$$
\begin{aligned}
\tilde{f}_{21}\left(x_{1}(t)\right)= & \frac{1}{J x_{1}}\left[-m g l \operatorname{sen}\left(x_{1}\right)\right. \\
& \left.-\lambda e^{-E x_{1}} e^{-E \frac{\pi}{2}}\left(x_{1}+\frac{\pi}{2}-\varpi\right)\right]
\end{aligned}
$$

Então, pode-se verificar em (35), que existe um problema de indeterminação de $\tilde{f}_{21}\left(x_{1}(t)\right)$ na simulação, quando for atribuído o valor zero para $x_{1}$, pois nesse caso, o denominador torna-se nulo. Assim, expandiu-se (35) (Teixeira et al., 2006a; Teixeira et al., 2006b; Gaino, 2009), por meio do software Matlab, em série de Taylor de quinta ordem, o que permite cancelar o termo $x_{1}$ no denominador de $\tilde{f}_{21}\left(x_{1}(t)\right)$ resolvendo o problema.

A Figura 5 mostra que a ordem utilizada para a série de Taylor representa satisfatoriamente a curva original no ponto de interesse. 
Figura 5 - Curvas da função $\tilde{f}_{21}\left(x_{1}(t)\right)$ exata e aproximação por série de Taylor de quinta ordem.

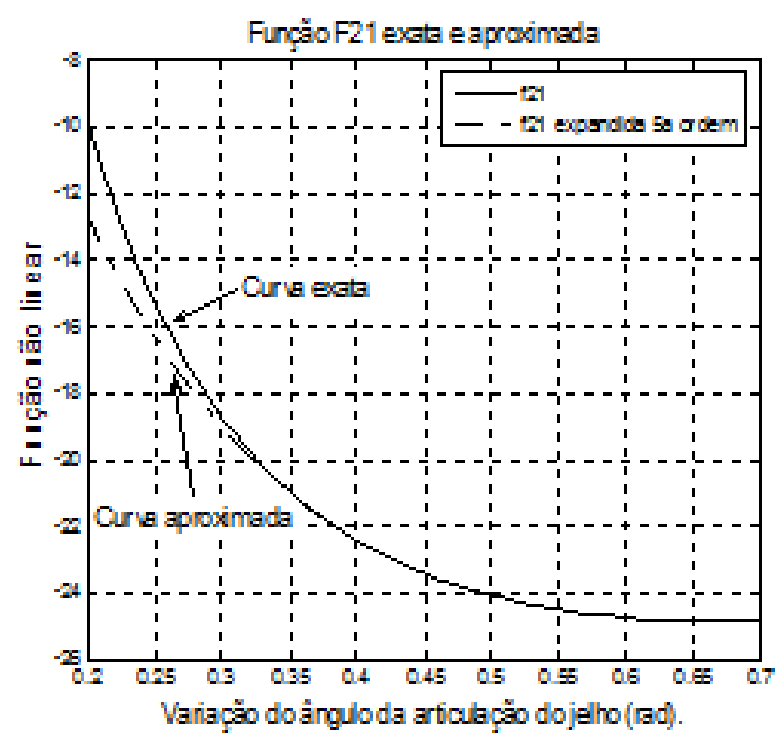

Fonte: Próprio Autor

O valor obtido para o ponto de $30^{\circ}(0.52 \mathrm{rad})$ foi:

$$
\tilde{f}_{21}\left(x_{1}(t)\right)=-24.3225
$$

\section{Resultados e Discussão}

Nesta seção, serão apresentados os resultados, na forma gráfica, obtidos a partir de diversas simulações. Considerando a planta descrita em (1) e (2), a representação em diagrama de blocos do sistema em malha fechada, utilizando o regulador LQR, é ilustrada na Figura 6.

A sistemática empregada para a seleção das matrizes pesos constituiu inicialmente de várias simulações em busca dos melhores valores de $\boldsymbol{Q}$. O critério utilizado foi relacionado com o tempo de estabilização. A configuração da matriz $\boldsymbol{R}$ foi a mesma em todos os modos.
Figura 6 - Projeto Regulador LQR.

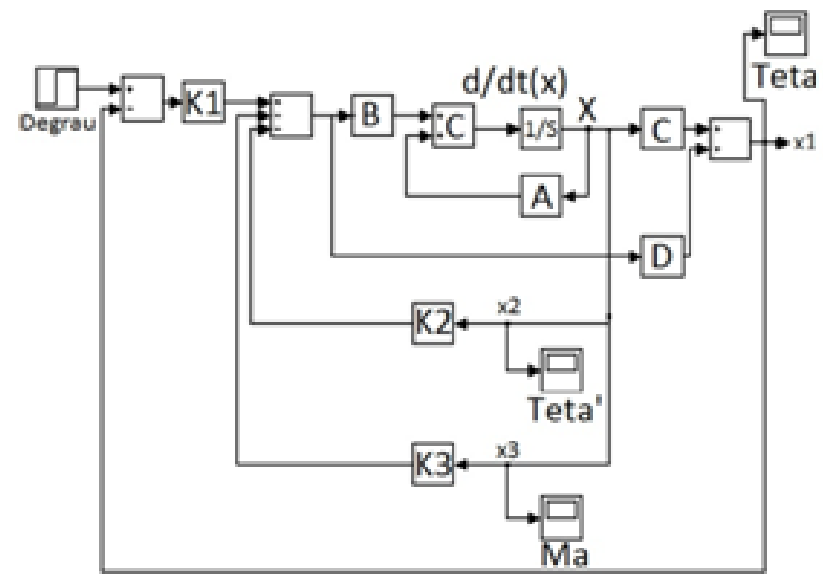

Fonte: Próprio Autor

As matrizes utilizadas para calcular a matriz ótima $K$ do regulador LQR, conforme a arquitetura de controle da Figura 6, são:

$$
\begin{aligned}
& A=\left[\begin{array}{ccc}
0 & 1 & 0 \\
-24.323 & -0.746 & 2.762 \\
0 & 0 & -1.051
\end{array}\right] \\
& \boldsymbol{B}=\left[\begin{array}{c}
0 \\
0 \\
4.469
\end{array}\right] \\
& C=\left[\begin{array}{lll}
1 & 0 & 0
\end{array}\right] \text {. } \\
& D=[0] .
\end{aligned}
$$

Adotou-se:

$$
\begin{gathered}
Q=\left[\begin{array}{ccc}
1 & 0 & 0 \\
0 & 0.5 & 0 \\
0 & 0 & 10^{-6}
\end{array}\right], \\
R=\left[10^{-6}\right] .
\end{gathered}
$$


A matriz ótima $\boldsymbol{K}$ foi obtida com o auxílio do software Matlab, que fornece a solução da equação de Riccati (22) para sistemas contínuos no tempo. Os ganhos $\boldsymbol{K}_{1}, \boldsymbol{K}_{2}$, e $\boldsymbol{K}_{3}$ obtidos a partir de $\boldsymbol{Q}$ e $\boldsymbol{R}$ são: $\boldsymbol{K}_{1}=996.19, \boldsymbol{K}_{2}=707.21$ e $\boldsymbol{K}_{3}=0.4329$.

As Figuras 7 a 9 mostram os resultados das simulações, realizadas em ambiente Matlab, do projeto do regulador (Figura 6), tendo como referência o degrau igual a $0.52 \mathrm{rad}$ ou $30^{\circ}$.

A Figura 7 representa o comportamento angular comum do joelho $\theta_{\dot{v}}$. A Figura 8 representa a velocidade angular comum do joelho $\dot{\theta}$. A Figura 9 mostra o torque ativo do joelho produzido pela estimulação elétrica $M_{a}$.

Figura 7 - Representação da variável $\theta_{v}$ tendo como referência o degrau igual a 0.52 .

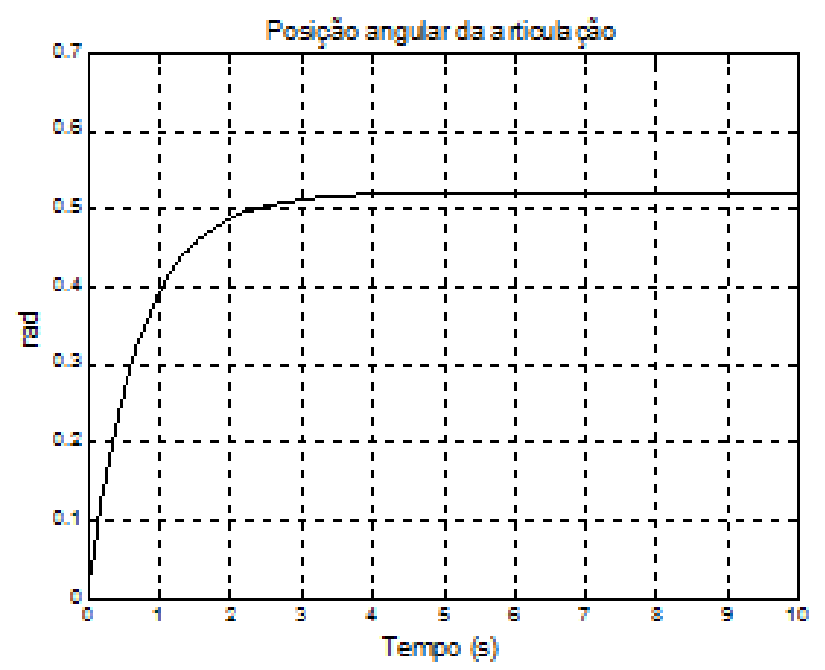

Fonte: Próprio Autor

Como pode ser observado na Figura 7, o ângulo $\theta_{v}$ estabilizou-se em $30^{\circ}(0,52 \mathrm{rad})$, atingindo a referência de entrada do degrau.

Na Figura 8, verifica-se que a velocidade angular do joelho $\dot{\theta}$ inicia-se próximo de $1,1 \mathrm{rad} / \mathrm{s}$ e reduzse indefinidamente, aproximando-se de zero em 2,5 segundos, aproximadamente.
Figura 8 - Representação da variável $\dot{\theta}_{v}$ tendo como referência o degrau igual a 0.52 .

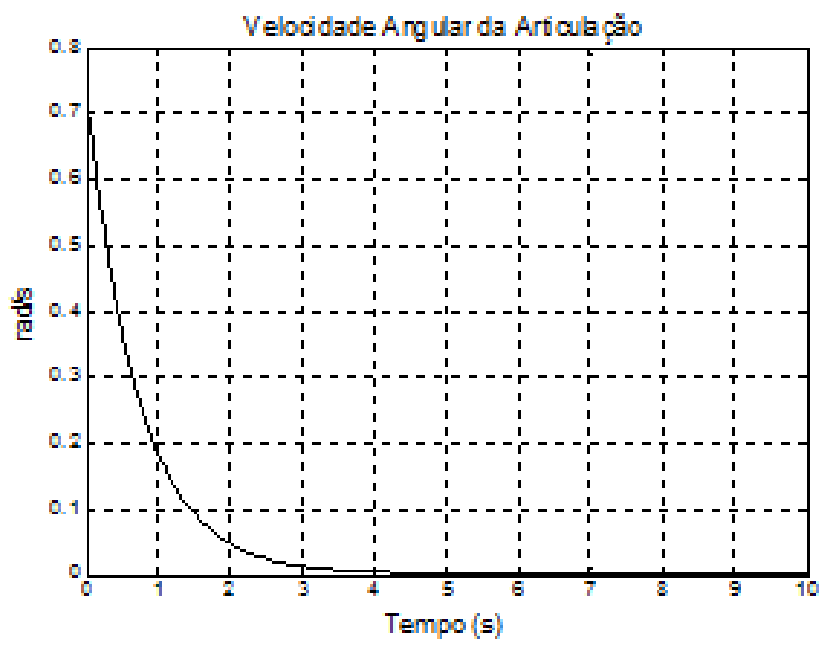

Fonte: Próprio Autor

A Figura 9 apresenta o torque ativo do joelho $M_{a}$ produzido pela estimulação elétrica, chegando a aproximadamente 4,6 $\mathrm{Nm}$.

Figura 9 - Representação da variável $M_{a}$ tendo como referência o degrau igual a 0.52 .

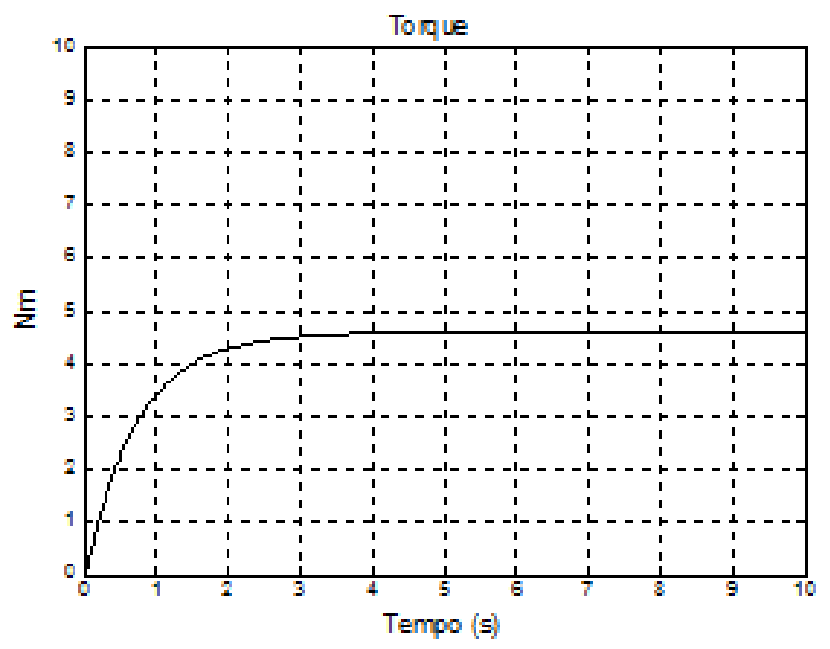

Fonte: Próprio Autor 
$\mathrm{Na}$ discussão desta metodologia, pode-se dizer que a mesma foi válida e que o sistema se estabilizou nos pontos de operação projetados. Esta metodologia é simples, por tratar-se de um sistema linear, com restrições de trabalho próximo ao ponto de operação. A aplicação de técnicas com controladores PID, que também são casos lineares, obteve bons resultados práticos, como mostrado em (Kozan, 2012). Para maior eficiência, também foram implementadas técnicas não lineares com modelos fuzzy Takagi-Sugeno (Gaino, 2009; Sanches, 2013). Em Gaino (2009), outros pontos de operação foram considerados: $47^{\circ}$ e $60^{\circ}$, como também, em Sanches (2013), no qual o projeto considerou o ponto de operação de $60^{\circ}$. Tanto o caso linear como o não linear responderam bem na implementação, o que foi projetado. O projeto considera um sistema com o paciente sentado. Futuramente, outras considerações devem ser feitas para que se obtenha a marcha humana e o estudo de outra modelagem, que considera o movimento das duas pernas. Estudos com variação paramétrica e rejeição de distúrbios podem ser considerados em trabalhos futuros, assim como o efeito de carregamento do sistema.

\section{Conclusões}

A teoria de controle foi utilizada no projeto do compensador necessário para compor as condições de desempenho exigidas em projeto. O LQR, embora seja uma técnica linear, mostrou-se por meio das simulações, um projeto de controle viável para aplicações no uso do FES em estímulos ao músculo quadríceps de pacientes paraplégicos, provocando movimentos na articulação do joelho.

As simulações realizadas com o software Matlab mostraram a eficiência do controlador LQR em malha fechada para um determinado ângulo da articulação do joelho, neste caso o complexo canela-tornozelo parte do repouso e estabiliza no ponto de operação desejado, ou seja, $30^{\circ}$ mostrando que o LQR é uma possível alternativa de controle da posição do complexo canela-tornozelo de pacientes paraplégicos utilizando eletroestimulação aplicada ao músculo quadríceps.

Com certeza técnicas não-lineares são mais precisas (Teixeira et al 2006a; Teixeira et al 2006b; Gaino, 2009), porém como em (Crago, Mortimer e Pecham, 1980), além deste artigo, vários modelos de sistemas lineares foram implementados por eles, confirmando que por meio de simulações (Gaino et al., 2008; Prado et al., 2008), a técnica de controle abordada neste artigo também pode ser implementada fisicamente.

\section{Referências}

ABBAS, J. J.; CHIZECK, H. J. Neural Network Control of Functional Neuromuscular Stimulation System: Computer Simulation Studies. IEEE Transactions on Biomedical Engineering, v. 42, n. 11, p. 1117-1127, 1995.

AGUIRRE, L. A. Introdução à identificação de sistemas: técnicas lineares e não-lineares aplicadas a sistemas reais. Belo Horizonte: Editora UFMG, 2000 .

CHANG, G.; LUH; J. LIAO, G.; LAI, J.; CHENG, C. A Neuro-Control System for the Knee Joint Position Control with Quadríceps Stimulation, IEEE Transactions on Rehabilitation Engineering, vol. 5. n. 1, p. 2-11, 1997.

CHANG, M.; WANG, K. Implementation of Fuzzy Control Over FES-Assisted Locomotion for CVA Patients. In: Proceding of the 20th annual International Conference of the IEEE Engineering in Medicine and Biology Society, vol. 20, n. 5, p. 2-11, 1998.

CHEN, Y.-L.; CHANG, W. WONG, M.-K.; TANG, F.-T.; CHEN, H.-I.; HUANG, C.-S.; KUO, T.-S. "A Treadmill Apparatus with a Computer-Assisted Functional Electrical Stimulation System by Foot Switch Controlled for Restoration of Ambulation in Stroke Patients". In: Proceedings - 19th International Conference - IEEE/EMBS, Chicago/ IL: October/1997. 
CHIZECK, H. J.; KOFMAN, L.; CRAGO, P. E.; WILHERE, G. F. Pulse-train controllers for functional neuromuscular stimulation. IEEE Transactions on Rehabilitation Engineering, San Diego, v. 14, n. 5, p. 628-639, 1983.

COVACIC, M. R.; TEIXEIRA, M. C. M., ASSUNÇÃO, E.; GAINO, R. LMI-Based Algorithm for Strictly Positive Real Systems with Static Output Feedback. Systems \& Control Letters v. 61, p. 521-527, 2012.

CRAGO, P. E.; MORTIMER, T.; PECHAM, P. H. Closed-Loop control of force during electrical stimulation of muscle. IEEE Transactions on Rehabilitation Engineering, San Diego, v. 27, n. 6, p. 306-312, 1980.

CRAMERI, R., M., COOPER, P., SINCLAIR P.J., BRYANT, G., WESTON, A. Effect of load during electrical stimulation training in spinal cord injury. Muscle \& Nerve, 29:01, p. 104-111, 2004

DELATORE, F. Controle multivariável em redes de trocadores de calor com bypasses. Tese de Doutorado, Escola Politécnica, Universidade de São Paulo, São Paulo, 2011.

FARIA, U. C. Implementação De Um Sistema De Geração De Marcha Para Pacientes Com Lesões Medulares. Tese (Doutorado) — Faculdade de Engenharia, Universidade Estadual Paulista, Ilha Solteira, 2006.

FERRARIN, M.; PEDOTTI, A. The relationship between electrical stimulus and joint torque: a dynamic model. IEEE Transactions on Rehabilitation Engineering, San Diego, v. 8, n. 3, p. 342-352, 2000.

GAINO, R. Controle de Movimentos de Pacientes Paraplégicos Utilizando Modelos Fuzzy T-S. Tese (Doutorado) — Faculdade de Engenharia, Universidade Estadual Paulista, Ilha Solteira, 2009.
GAINO, R.; SILVA, T. I.; SANCHES, M. A. A.; FREITAS, R. L. B.; SATO, F. H.; SANTOS; E. A.; CARVAlHO, A. A. C.; TEIXEIRA, M. C. M.; ASSUNÇÃO, E.; MACHADO, E. R. M. D. Controle Digital Para FES no Estímulo do Músculo Quadríceps em Pacientes Paraplégicos In: 6th Congresso Temático de Dinâmica, Controle e Aplicações, 2007, São José do Rio Preto, p. 764$771,2007$.

GAINO, R.; TEIXEIRA, M. C. M. ; CARDIM, R. ; CARVALHO A. A. ; ASSUNÇÃO, E. ; SANCHES, M. A. A. Controle Não-Linear Fuzzy TakagiSugeno do Movimento de Paraplégicos Utilizando Acelerômetros. In: Anais do XXI Congresso Brasileiro de Engenharia Biomédica. Salvador : Grupo GT5, p. 1254-1257, 2008.

GAINO, R.; TEIXEIRA, M. C. M.; CARVALHO,A. A.; ASSUNÇÃO, E.; CARDIM, R.; SANCHES, M. A. A.; COVACIC, M. R . Realimentação derivativa e modelo fuzzy Takagi-Sugeno para controle da articulação do joelho de pacientes paraplégicos com o uso de acelerômetros. Revista Brasileira de Engenharia Biomédica, v. 27, n. 2, p. 67-78, 2011.

HANDA, T.; TAKAHASHI, H.; SAITO, C.; ICHIE, M.; HANDA, Y.; KAMEYAMA, J.; HOSHIMIYA, N. "Development of an FES System Controlled by EMG Signals". Annual International Conference of the IEEE Engineerring in Medicine and Biology Society, Vol. 12, No 5, 1990.

HATZE, H . Myocybernetic control models of skeletal muscle. Pretoria: University of South Africa Press, 1981.

HERZOG, W.; NIGG, B. M (1999). Biomechanics of musculoskeletal system. Chichester, England: Wiley \& Sons Ltd.

HILL, A. V . The heat of shortening and the dynamic constant of muscle. Proceedings of the Royal Society, B, n. 126, p. 136-195, 1938.

HUXLEY, A. F. Muscle structure and theories of contraction. Progress and Biophysical Chemistry, Elmsford, n. 7, p. 255-318, 1957. 
IQBAL, K.; MUGHAL, A. Active Control vs. Passive Stiffness in Posture and Movement Coordination. In: IEEE International Conference on Systems, Man and Cybernetics, Montréal, Canada. P. 3367-3372, 2007.

KAILATH, T. Linear Systems. USA: Prentice Hall Inc., 1980.

KOZAN, R. F. Controle da posição da perna de pessoas hígidas utilizando um controlador PID. Dissertação (Mestrado) — Faculdade de Engenharia, Universidade Estadual Paulista, Ilha Solteira, 2012.

MARTIN, P. S. Pesquisadores da Unicamp desenvolveram aparelhos para recuperar paraplégicos. Jornal da Unicamp, Campinas, p. 57-61, 1999.

MATJACIC, Z.; BAJD, T. Arm-free Paraplegic Standing - Part I: Control Model Synthesis and Simulation. IEEE Transactions on Rehabilitation Engineering, Vol. 6, n. 2, pp. 125-138, 1998.

OGATA, K . Engenharia de Controle Moderno. São Paulo: Pearson Prentice Hall 5a edição, 2010

PRADO, T. A. ; GAINO, R. ; CARVALHO A. A. ; PEREIRA, M. C. ; BATISTA, E. A. ; CARNEIRO, A. M. Desenvolvimento de Uma Ferramenta Para Projeto de Controladores PID Aplicados em Estimulação Neuromuscular Funcional. In: Anais do XXI Congresso Brasileiro de Engenharia Biomédica. Salvador : Grupo GT5, p. 891-894, 2008.

PETERSON, D. K.; CHIZECK, H. J. Linear Quadratic Control of a Loaded Agonist-Antagonist Muscle Pair. IEEE Transactions on Biomedical Engineering, Vol. BME-34, No. 10, pp. 790 - 796, 1987.

RIENER, R.; FUHR, T. Patient-driven control of FES-supported standing up: a simulation study. IEEE Transaction on Rehabilitation Engineering, San Diego, v. 6, n. 2, p. 113-124, 1998.
ROBINSON, A. J. "Eletrofisiologia Clínica: Eletroterapia, e Teste Fisiológico”. $2^{\circ}$ ed, Armed Editora: Porto Alegre/RS, 2001.

ROSA FILHO, J. E. A. Contribuições de Controle Ótimo. Trabalho de Conclusão de Curso, Universidade Estadual de Londrina, 2011.

SANCHES, M. A. A. Sistema eletrônico para geração e avaliação de movimentos em paraplégicos. Tese (Doutorado) — Faculdade de Engenharia, Universidade Estadual Paulista, Ilha Solteira, 2013.

SANCHES, M. A. A.; GAINO, R. CARVALHO A. A.; TEIXEIRA, M. C. M. ; PONTES, W. ; DOS SANTOS, E. A.; PRADO, T. A.; FREITAS, R. L. B. Projeto de Controladores digitais utilizando microcontroladores e o software Isis Proteus. In: XVIII Congresso Brasileiro de Automática, Bonito MS. 12 a 16-setembro-2010. Campo Grande MS : opec-eventos. p. 4752-4756, 2010.

SANCHES, M. A. A.; GAINO, R.; KOZAN, R. F.; TEIXEIRA, M. C. M.; CARVALHO, A. A.; COVACIC, M. R.; ALVES, C. A.; URBAN, M. F. R.; JUNQUEIRA, M. V. N.; CARDIM, R.; ASSUNÇÃO, E.; GENTILHO JUNIOR, E. Digital controllers design considering hardware constraints: Application in a paraplegic patient, Brazilian Journal of Biomedical Engineering, UberlândiaBR, v. 30, n. 3, 11 páginas, 2014. (a ser publicado)

SILVA, T. I. Implementação de um Sistema para Geração e Avaliação de Movimentos em Pacientes Hemiplégicos. Tese (Doutorado) - Faculdade de Engenharia, Universidade Estadual Paulista, Ilha Solteira, 2007.

TEIXEIRA, M. C. M.; DEAECTO, G. S.; GAINO, R.; ASSUNÇÃO, E.; CARVALHO, A. A.; FARIA, U. C. Design of a fuzzy Takagi-Sugeno controller to vary the joint knee angle of paraplegic patients. Lectures Notes in Computer Science Springer Berlin/Heidelberg, v. 4234, n. 3, p. 118-126, 2006a. 
TEIXEIRA, M. C. M.; DEAECTO, G. S.; GAINO, R.; ASSUNÇÃO, E.; CARVALHO, A. A.; FARIA, U. C. Projeto de um controlador fuzzy TakagiSugeno para variar o ângulo da articulação do joelho. In: Anais do XVI Congresso Brasileiro de Automática, CBA, Salvador, p. 2287-2292, 2006 b.

WINTER, D. A. Biomechanics and motor control of human movement. New York: John Wiley Sons, Inc, 1990.

YU, W.; MARUSIHI, M.; YOKOI, H.; MANO, Y.; KAKAZU, Y. "An Architecture for Adaptive Functional Electrical Stimulation for Hemiplegic Lower Limb Activities", In: Proceedings of the 1999 IEEE International Workshop on Roobot and Human Interaction, Pisa, Italy: September, 1999.

Recebido em 26 Agosto, 2012 - Received on August 26, 2012

Aceito em 15 Julho, 2014 - Accepted on July 15, 2014 
